\title{
BETHE ANSATZ AND THE GEOGRAPHY OF RIGGED STRINGS
}

\author{
TADEUSZ LULEK \\ Rzeszów University of Technology \\ Powstańców Warszawy 6, 35-959 Rzeszów, Poland \\ E-mail: tadlulek@prz.edu.pl
}

\begin{abstract}
We demonstrate the way in which composition of two famous combinatorial bijections, of Robinson-Schensted and Kerov-Kirillov-Reshetikhin, applied to the Heisenberg model of magnetic ring with spin $1 / 2$, defines the geography of rigged strings (which label exact eigenfunctions of the Bethe Ansatz) on the classical configuration space (the set of all positions of the system of $r$ reversed spins). We point out that each $l$-string originates, in the language of this bijection, from an island of $l$ consecutive reversed spins. We also explain travel of $l$-strings along orbits of the translation group of the ring.
\end{abstract}

1. Introduction. Bethe Ansatz (BA) [1-4] provides the exact solution of the eigenproblem of the Heisenberg Hamiltonian for the ring of $N$ magnetic nodes, each node with the spin $1 / 2$, with the nearest-neighbour isotropic interaction. Existence of this solution is an effect of integrability of such a quantum model of a magnet. Within BA approach, each eigenstate of the Heisenberg Hamiltonian is a vector in an appropriate subspace $\mathcal{H}^{(r)} \subset \mathcal{H}$, spanned by all such magnetic configurations on the ring which have $r$ reversed spins. Since the original paper [1] of Bethe it was evident that such an eigenstate can be interpreted as a collection of $l$-strings, bound states of $l$ reversed spins, each $l$-string equipped with a "rigging", i.e. a definite quasimomentum, expressed as an integer related to an irreducible representation of the cyclic group $C_{N}$, the translational symmetry group of the magnetic ring. This interpretation was raised to the level of a classification scheme by Kerov, Kirillov and Reshetikhin (KKR) [5], who gave a formal definition of some combinatorial objects, rigged string configurations of various types, and proved that, in particular, some specified sets of such rigged strings provide a unique labeling of all exact BA eigenstates. Moreover, they have pointed out an explicit construction of a bijection

2000 Mathematics Subject Classification: Primary 20C30; Secondary 70G10.

Key words and phrases: Robinson-Schensted algorithm, rigged strings, Bethe Ansatz, Weyl duality.

The paper is in final form and no version of it will be published elsewhere. 
between the set of pairs of standard Young and Weyl tableaux of the same shape, and some corresponding sets of rigged strings. This KKR bijection paves the way for a purely combinatorial settlement of BA, since it can be composed with the Robinson-Schensted (RS) algorithm [6-9], providing thus an immediate bijection between the set of all magnetic configurations, the initial basis of the space $\mathcal{H}$, and the rigged strings, the basis of exact BA eigenstates $[5,10]$.

Each subspace $\mathcal{H}^{(r)} \subset \mathcal{H}$ with the fixed number $r$ of spin deviations, $0 \leq r \leq N$, can be seen as the space of all quantum states of the system of $r$ objects, hereafter referred to as Bethe pseudoparticles. These objects are indistinguishable, have hard core (i.e. two or more of them cannot occupy the same site), and move on the magnetic ring $\tilde{N}=\{j=1,2, \ldots, N\}$ by jumps to nearest neighbour sites $(j \mapsto(j \pm 1) \bmod N)$ due to transitions imposed by the Heisenberg Hamiltonian. Thus the set $\tilde{N}$ plays the role of the classical configuration space of a single Bethe pseudoparticle, and the corresponding classical configuration space for the system $\tilde{r}=\{\alpha=1,2, \ldots, r\}$ of pseudoparticles is

$$
Q^{(r)}=\left(\tilde{N}^{\times r} \backslash D(N, r)\right) / \Sigma_{r},
$$

that is, the generic stratum of the action of the symmetric group $\Sigma_{r}$ (the indistinguishability group for this case) on the $r$-th Cartesian power of $\tilde{N}$, with $D(N, r) \subset \tilde{N}^{\times r}$ being the subset of all coincidences, or "the fat diagonal" of $\tilde{N}^{\times r}$ (see, e.g., [11] and references therein). We use here the term "the classical configuration space" as a characteristic of the classical counterpart of a quantum system, the system of $r$ Bethe pseudoparticles on the ring $\tilde{N}$, so that the linear closure

$$
l c_{\mathbb{C}} Q^{(r)}=\mathcal{H}^{(r)}
$$

of the set $Q^{(r)}$ over the field $\mathbb{C}$ of complex numbers is identified with the space $\mathcal{H}^{(r)}$, and the set $Q^{(r)}$ is the basis of all possible "positions" of the system along the Schrödinger quantization scheme. In particular, $\mathcal{H}^{(1)}=l c_{\mathbb{C}} \tilde{N}$ is the space of all quantum states of a single magnon.

Clearly, the classical configuration space $Q^{(r)}$ can be identified with the set

$$
Q^{(r)}=\left\{\mathbf{j}=\left(j_{1}, j_{2}, \ldots, j_{r}\right) \mid 1 \leq j_{1}<j_{2}<\cdots<j_{r} \leq N\right\}
$$

of all magnetic configurations with $r$ spin deviations on the ring $\tilde{N}$. Moreover, the set $Q^{(r)}$ can be embedded, for an arbitrary $N \geq 2 r$ (we restrict in the sequel to the case $r \leq N / 2$, having in mind the obvious particle-hole symmetry), into a real manifold $\mathcal{M}^{(r)}$ with some boundaries. The generic part of the manifold $\mathcal{M}^{(r)}$ is locally $r$-dimensional, and is related to cases when all $r$ Bethe pseudoparticles are "remote", i.e. separated by some nodes occupied by the spin "+", whereas $F$-dimensional boundaries, $1 \leq F<r$, correspond to cases when a magnetic configuration $\mathbf{j} \in Q^{(r)}$ consists of exactly $F$ islands of reversed spins [10, 12].

Existence of the composed bijection $R S K K R=K K R \circ R S$ which maps $Q^{(r)}$ onto the BA eigenstate basis of the space $\mathcal{H}^{(r)}$, establishes a geography of rigged strings of $\mathrm{BA}$ on the classical configuration space. We aim here to describe this geography in some detail. In particular, we shall point out how $l$-strings originate from those boundaries of the manifold $\mathcal{M}^{(r)}$, whose dimension $F=l$. 
2. Bethe Ansatz in terms of the duality of Weyl. An adequate framework for BA is provided by the famous duality of Weyl between the symmetric group $\Sigma_{N}$ and the unitary group $U(n)$ [13], acting in a natural way in the space $\left(\mathbb{C}^{n}\right)^{\otimes N}$. The dual integers, $n$ and $N$, in the case of BA with spin $1 / 2$, denote the dimension $n=2$ of the single-node space $h=\mathbb{C}^{2}$, and the number of magnetic nodes, respectively. Correspondingly, the dual sets are alphabets of spins $\tilde{2}=\{+,-\}$, and of nodes $\tilde{N}$. The set

$$
\tilde{2}^{\tilde{N}}=\{f: \tilde{N} \rightarrow \tilde{2}\}
$$

of all magnetic configurations spans unitarily the space

$$
\mathcal{H}=l c_{\mathbb{C}} \tilde{2}^{\tilde{N}} \cong \prod_{j \in \tilde{N}} \otimes h_{j}
$$

of all quantum states of the magnet, with $h_{j}=l c_{\mathbb{C}} \tilde{2} \cong \mathbb{C}^{2}$ being the space related to the node $j \in \tilde{N}$. $\mathcal{H}$ is the carrier space of two dual actions, $A: \Sigma_{N} \times \mathcal{H} \rightarrow \mathcal{H}$ and $B: U(2) \times \mathcal{H} \rightarrow \mathcal{H}$, defined by formulas

$$
A(\sigma)=\left(\begin{array}{c}
f \\
f \circ \sigma^{-1}
\end{array}\right), f \in \tilde{2}^{\tilde{N}}, \sigma \in \Sigma_{N},
$$

and

$$
B(u)=\left(\begin{array}{c}
f \\
B(u) f
\end{array}\right), \quad f \in \tilde{2}^{\tilde{N}}, u \in U(2),
$$

where $f \circ \sigma^{-1} \in \tilde{2}^{\tilde{N}}$ is the composition of mappings $f$ and $\sigma^{-1}$, and

$$
B(u) f \equiv B(u)\left|i_{1} i_{2} \ldots i_{N}\right\rangle=\sum_{i_{1}^{\prime}, i_{2}^{\prime}, \ldots, i_{N}^{\prime}} u_{i_{1}^{\prime} i_{1}} u_{i_{2}^{\prime} i_{2}} \ldots u_{i_{N}^{\prime} i_{N}}\left|i_{1}^{\prime} i_{2}^{\prime} \ldots i_{N}^{\prime}\right\rangle
$$

is the multilinear extension of the defining action of the unitary group $U(2)$ in the space $h$.

The key point of the duality of Weyl is that these two dual actions commute, i.e.

$$
[A(\sigma), B(u)]=0, \quad \sigma \in \Sigma_{N}, \quad u \in U(2),
$$

which implies a quantum-mechanical interpretation that appropriate operators "can be measured simultaneously". This means that there exists a basis in the space $\mathcal{H}$ which is irreducible under both actions. Let

$$
A=\sum_{\lambda \in D_{W}(N)} m\left(A, \Delta^{\lambda}\right) \Delta^{\lambda}
$$

and

$$
B=\sum_{\lambda \in D_{W}(N)} m\left(B, D^{\lambda}\right) D^{\lambda}
$$

denote the decomposition of actions $A$ and $B$, treated as linear representations, into appropriate irreps, $\Delta^{\lambda}$ of $\Sigma_{N}$ and $D^{\lambda}$ of $U(2)$, with multiplicities $m\left(A, \Delta^{\lambda}\right)$ and $m\left(B, D^{\lambda}\right)$, respectively, and $D_{W}(N)$ denoting the set of all partitions of $N$ into at most two parts, so that the partition $\lambda \vdash N$ has the form

$$
\lambda=\left(N-r^{\prime}, r^{\prime}\right), \quad 0 \leq r^{\prime} \leq N / 2 .
$$


Then the space $\mathcal{H}$ decomposes into mutually orthogonal subspaces $\mathcal{H}^{\lambda}$, referred to as sectors,

$$
\mathcal{H}=\sum_{\lambda \in D_{W}(N)} \oplus \mathcal{H}^{\lambda}
$$

such that

$$
\left.A\right|_{\mathcal{H}^{\lambda}}=m\left(A, \Delta^{\lambda}\right) \Delta^{\lambda}
$$

and

$$
\left.B\right|_{\mathcal{H}^{\lambda}}=m\left(B, D^{\lambda}\right) D^{\lambda},
$$

so that the multiplicity of $\lambda$ in each action is equal to the dimension of the corresponding irrep of the dual action. At the level of bases, each sector is spanned unitarily by the set $S Y T(\lambda) \times W T(\lambda)$, where $S Y T(\lambda)$ is the set of all standard Young tableaux of the shape $\lambda$ on the alphabet $\tilde{N}$ of nodes, and $W T(\lambda)$ is the set of all Weyl (semistandard) tableaux of the same shape on the alphabet $\tilde{2}$ of spins, i.e.

$$
\mathcal{H}^{\lambda}=l c_{\mathbb{C}}(S Y T(\lambda) \times W T(\lambda)) .
$$

We refer to this basis, written as

$$
R S(N)=\left\{|\lambda t y\rangle \mid \lambda \in D_{W}(N), t \in W T(\lambda), y \in S Y T(\lambda)\right\},
$$

as to the irreducible basis of the Weyl duality in the space $\mathcal{H}$ of quantum states of the magnet. It is worth to observe that the basis $R S(N)$ provides a complete classification of basis states in $\mathcal{H}$. Another important observation is that the sets $\tilde{2}^{\tilde{N}}$ and $R S(N)$ are respectively the source and target for the famous Robinson-Schensted bijection $R S$ : $\tilde{2}^{\tilde{N}} \rightarrow R S(N)$ [6-9]. The set $R S(N)$ serves also as the source of the $K K R$ bijection.

Elements $|\lambda t y\rangle$ of the irreducible basis of the Weyl duality are not, in general, eigenstates of the Heisenberg Hamiltonian, since the group $\Sigma_{N}$ is not the symmetry group of the magnet. However, each vector $|\lambda t y\rangle$ provides two important quantum numbers, the total spin

$$
S=N / 2-r^{\prime}
$$

of the magnet, determined by the partition $\lambda=\left(N-r^{\prime}, r^{\prime}\right)$, and its $z$-projection

$$
M=N / 2-r,
$$

derived from the weight $w t(t)=(N-r, r)$ of the Weyl tableau $t$.

3. The dynamics of BA. Isotropy of the Heisenberg Hamiltonian implies the spherical symmetry, so that the total spin $S$ and its $z$-projection $M$ are exact quantum numbers of the model. Thus the number $r$ of Bethe pseudoparticles is also a constant of motion, and the dynamics of the magnet can be considered for each $\mathcal{H}^{(r)}$ subspace separately. The classical configuration space $Q^{(r)}$, i.e. the initial basis for quantum calculations, can be seen as the orbit of the action $A$, generated from an arbitrary magnetic configuration with the weight

$$
\mu=\{N-r, r\}, \quad 0 \leq r \leq N / 2,
$$

i.e.

$$
Q^{(r)}=\left\{f \circ \sigma^{-1} \mid \sigma \in \Sigma_{N}\right\}
$$


with $|f\rangle=|\underbrace{+\cdots+}_{N-r} \underbrace{-\cdots-}_{r}\rangle$. Accordingly, the restriction of $A$ to $Q^{(r)}$ is a transitive representation of the group $\Sigma_{N}$, denoted by

$$
\left.A\right|_{Q^{(r)}}=R^{\mu}, \quad \mu=\{N-r, r\} .
$$

The model requirement of the nearest neighbour interaction yields

$$
\hat{H}|\mathbf{j}\rangle=\sum_{\mathbf{j}^{\prime} \in Q_{\mathbf{j}}^{(r)}}\left(\left|\mathbf{j}^{\prime}\right\rangle-|\mathbf{j}\rangle\right), \mathbf{j} \in Q^{(r)},
$$

where $\hat{H} \in E n d \mathcal{H}$ is the Heisenberg Hamiltonian, and $Q_{\mathbf{j}}^{(r)}$ is the set of all magnetic configurations in $Q^{(r)}$ which differ from $\mathbf{j} \in Q^{(r)}$ only in one component, say $\alpha_{0} \in \tilde{r}$, and only by the distance 1 , i.e.

$$
j_{\alpha}^{\prime}= \begin{cases}\left(j_{\alpha_{0}}^{\prime} \pm 1\right) \bmod N & \text { for } \alpha=\alpha_{0}, \\ j_{\alpha} & \text { for } \alpha \in \tilde{r} \backslash\left\{\alpha_{0}\right\} .\end{cases}
$$

In this way, the dynamics of the Heisenberg Hamiltonian $\hat{H}$ is consistent with the geometry of the manifold $\mathcal{M}^{(r)}$ into which $Q^{(r)}$ is embedded. In short, $\hat{H}$ acts locally in $Q^{(r)}$.

Eigenstates of the Hamiltonian $\hat{H}$ in the space $\mathcal{H}^{(r)}$ have all the same eigenvalue $M=$ $N / 2-r$, but can differ by the total spin $S$, in accordance with the Kostka decomposition

$$
R^{\mu}=\sum_{\lambda \in D_{W}(N)} K_{\lambda \mu} \Delta^{\lambda}
$$

which in our case simplifies to

$$
R^{(N-r, r)}=\sum_{r^{\prime}=0}^{r} \Delta^{\left(N-r^{\prime}, r^{\prime}\right)} .
$$

The term $r^{\prime}=r$ corresponds to the highest weight $(S=M)$ eigenstates, whereas for $r^{\prime}<r$ one has $S>M$. In particular, $r^{\prime}=0$ corresponds to $S_{\max }=N / 2$, the unique state in each $\mathcal{H}^{(r)}$, which is degenerated with the ferromagnetic vacuum configuration $|+\cdots+\rangle$. More generally, lower weight eigenstates $(S>M)$ in $\mathcal{H}^{(r)}$ are all degenerated in energy with some corresponding eigenstates belonging to subspaces with smaller number of Bethe pseudoparticles. Thus in the extreme case

$$
r_{\max }= \begin{cases}N / 2 & \text { for } N \text { even } \\ (N-1) / 2 & \text { for } N \text { odd }\end{cases}
$$

(i.e. the maximal not beyond the equator), the corresponding space $\mathcal{H}^{\left(r_{\max }\right)}$ contains eigenstates from each energy level and each sector $\mathcal{H}^{\lambda}$.

4. The bijection between magnetic configurations and rigged strings. Perhaps the best visualisation of rigged strings in combinatorial description of the announced bijection can be provided in terms of paths. Let us consider the set of all points in the $(j, l)$-plane, given by vectors with non-negative integral coefficients, with $j \geq l$. A path is defined recursively with respect to $j=1,2, \ldots, N$, as a continuous line consisting of consecutive segments such that (i) the segment of the node $(j-1) \in \tilde{N}$ joins the point 
$(j-1, l)$, given by the recursive assumption, to either $(j, l+1)$ (step up), or $(j, l-1)$ (step down), (ii) the path starts at the point $(0,0)$, so that the first segment ends at $(1,1)$. We denote a path $p$ as a sequence of steps, $p=\left(p_{1}, p_{2}, \ldots, p_{N}\right), p_{j} \in\{\operatorname{up}, \operatorname{down}\}, j \in \tilde{N}$, in particular $p_{1}=$ up. An example of a path is given in Fig. 1.

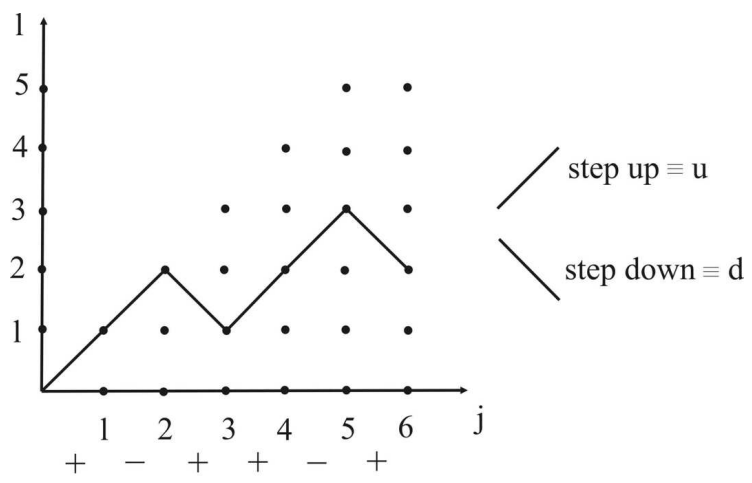

Fig. 1. An example of a path, for $N=6, p=(u, u, d, u, u, d)$

A typical single $l$-string is presented by the path in Fig. 2. The path encloses a single pyramid of height $l$ and base $2 l$. Moreover, this path has two straight lines, consisting of $L$ steps up to the left of the pyramid, and $N-L-2 l$ steps up to the right of it. These two straight lines are referred to as the sea of $l$-holes. The location of the $l$-string in the sea of holes is specified by the integer $L$, referred to as the rigging of the $l$-string, and satisfying $0 \leq L \leq N-2 l$.

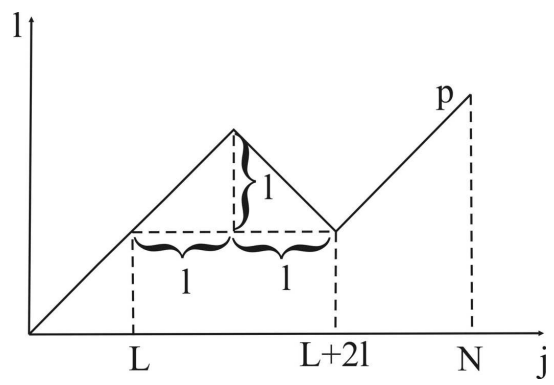

Fig. 2. A single $l$-string has the shape of a pyramid of height $l$ and base $2 l$, located in the sea of $l$-holes (the left and right straight lines on the path $p$ ) according to the rigging $L$

It is an interesting and even somehow intriguing point that a complete classification of exact BA eigenstates of the Hamiltonian $\hat{H}$ of Eq. (23) has been reached by an interpretation of the $l$-string presented in Fig. 2 as a bound state of $l$ consecutive Bethe pseudoparticles, with the rigging $L$ characterising the quasimomentum of this state. More specifically, one can interpret combinatorially the $l$-string as a composite system on the magnetic ring, which consists of $l$ Bethe pseudoparticles located on consecutive nodes, and coupled to the next consecutive $l$ nodes to the right, occupied by the spin " + ". In 
other words, the $l$-string is represented by the pyramid on the path $p$ of Fig. 2 , and thus is characterised by the height $l$ and the length $2 l$. An isolated $l$-string can exist in $N-2 l+1$ different kinematical states, labeled by the rigging $L, 0 \leq L \leq N-2 l$. In other words, the arena for such an $l$-string is the chain $\tilde{N}$ with distinguished initial and terminal node ( $j=1$ and $j=N$, respectively), and the $l$-string is a hard-core object of size $2 l$, placed somewhere into this chain according to the rigging $L$.

One can replace the chain $\tilde{N}$, the regular orbit of the cyclic group $C_{N} \subset \Sigma_{N}$, i.e. the translation symmetry group of the system, by the Brillouin zone

$$
B=\left\{k=0, \pm 1, \pm 2, \ldots,\left\{\begin{array}{ll} 
\pm(N / 2-1), N / 2 & \text { for } N \text { even } \\
\pm(N-1) / 2 & \text { for } N \text { odd }
\end{array}\right\}\right.
$$

which is the set of labels of all irreps $\Gamma_{k}$ of $C_{N}\left(\Gamma_{k}(j)=\exp (2 \pi i k j / N), j \in \tilde{N}\right)$, and thus the set of all admissible quasimomenta of the magnet. In this setting, the hard-core effect of the $l$-string implies that this object cannot take on any value of quasimomentum from the Brillouin zone $B$, and the forbidden values of quasimomentum are placed symmetrically near the center $k=0 \in B$, so that

$$
B=B_{f} \cup B_{a},
$$

where the forbidden part is

$$
B_{f}=\{k=0, \pm 1, \pm 2, \ldots, \pm(l-1)\},
$$

whereas the admissible region $B_{a}=B \backslash B_{f}$ is the range of the rigging $L$, such that

$$
k=(l+L) \bmod B
$$

is the quasimomentum of the single $l$-string.

A single isolated $l$-string is a very special case of an exact BA eigenstate, but it covers already many essential features of a general case, at least in the combinatorial setting. For example the path in Fig. 1 corresponds to two 1 -strings, with the riggings $L_{1}=1$ and $L_{2}=2$. In general, each path $p$ defines, by its shape, the content of strings, given as a partition $\nu \vdash r^{\prime}$ whose each row of length $l$ corresponds to an $l$-string, so that

$$
\sum_{l} l \nu_{l}=r^{\prime}, \quad 0 \leq r^{\prime} \leq r
$$

where $\nu_{l}$ is the total number of $l$-strings, $r^{\prime}=N / 2-S$ is the number of Bethe pseudoparticles bound into strings (and thus $r-r^{\prime}$ Bethe pseudoparticles are unbounded, and placed at the rightmost part of the sea of holes). Each path $p$ defines also, in addition to the content $\nu$ of strings, the position of each string on the chain $\tilde{N}$, specified by its rigging. The string content $\nu$, taken together with the set $\mathcal{L}$ of riggings, defines the rigged string configuration $\nu \mathcal{L}$, corresponding to the path $p$. The latter is used to label exact BA eigenstates.

The rigged string configuration $\nu \mathcal{L}$ can be easily derived from the path $p$ in cases when the corresponding pyramids do not overlap, as e.g. in Fig. 1. In general, however, determination of the rigged string configuration $\nu \mathcal{L}$ from a given path $p$ is a complex task which requires the rules of navigation, described by Warnaar [14] (cf. also Welsh [15, 16]). The essential difficulty in resolution of a given path $p$ into rigged strings $\nu \mathcal{L}$ consists in a 
proper selection of pyramids when they overlap. To this end, one applies two navigation rules: (i) two strings of the same height $l$ are mutually impenetrable, i.e. they behave like hard-core objects, (ii) for $l<l^{\prime}$, the (smaller) $l$-string can slide over both slopes of the (larger) $l$-string, until the peaks of representing pyramids coincide. Thus, e.g., the string content $\nu=\{31\}$ for the chain of $N=8$ nodes is realised only for five paths, shown in Fig. 3. The first and the last path present the adjacent, but not overlapping strings, whereas the remaining three correspond to the stages of sliding of the smaller 1-string on both slopes of the larger 3 -string. It is worth to observe that a location of a string on the chain $\tilde{N}$ by the peak of the corresponding pyramid is unique with the exception of the third path, where both peaks have the same $l$-level.

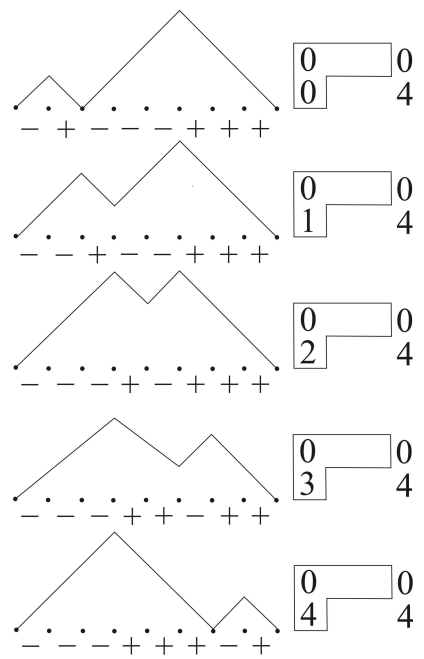

Fig. 3. Sliding of a 1-string over both slopes of a 3-string

Each path $p$ in the $(j, l)$-plane looks thus like a contour of a mountain ridge, consisting of $N-r$ steps up and $r$ down joined together, with local cuspidal maxima and minima. Clearly, such a path uniquely decomposes into two types of segments of consecutive steps: (i) straight diagonal lines, i.e. steps up only, (ii) multipyramids, with the same number $A$ of steps up and down, such that the height $l(j)$ takes on a minimal value $l_{0}$ at the initial node $j_{0}$ and terminal $j_{0}+2 A-1$, and some larger values at all intermediate nodes. Each segment of the type (i) represents a connected part of the sea of holes (for each string), whereas each multipyramid corresponds to a number of overlapping strings, with the total number of strings equal to that of peaks of the multipyramid.

The range of riggings is determined by the hard-core effects, described by the navigation rules, and depends on the length of a string. Clearly, for each string of the maximal length (for a given content $\nu$ ), this range is $N-2 r^{\prime}$, the sum of lengths of the segments of sea of holes on appropriate paths. Each string of a smaller length acquires additional locations due to sliding on slopes of larger pyramids. These hard-core effects can be easily deduced from the Young diagram for the partition $\nu \vdash r^{\prime}$ of the content of strings. Let 
$Q_{l}$ be the number of boxes in the first $l$ columns of the partition $\nu$, i.e.

$$
Q_{l}=\sum_{l^{\prime}} m_{l^{\prime}} \min \left(l, l^{\prime}\right)
$$

(cf. Fig. 4).

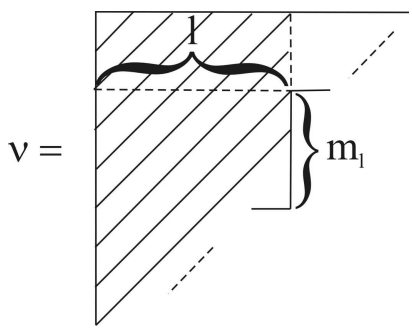

Fig. 4. The hard-core effect in counting $l$-holes. $Q_{l}$ is the number of boxes in the shadowed part which encloses the first $l$ columns of the Young diagram $\nu \vdash r^{\prime}$ of the string content (cf. Eq. $(33))$.

Then the integer

$$
P_{l}=N-2 Q_{l}
$$

is the upper bound for the rigging of an $l$-string, and the range for the collection of $m_{l}$ $l$-strings is specified by

$$
0 \leq L_{l v} \leq L_{l v^{\prime}} \leq P_{l}, \quad 1 \leq v<v^{\prime} \leq m_{l} .
$$

It implies that the total number of riggings for a given string content $\nu$ is

$$
|z(\nu)|=\prod_{l}\left(\begin{array}{c}
P_{l}+m_{l} \\
m_{l}
\end{array}\right) .
$$

In this way, each path of length $N$ (i.e. consisting of $N$ steps), which starts at $(0,0)$ and terminates at $(N, r)$, describes a rigged string $\nu \mathcal{L}$, with $\nu \vdash r^{\prime}, 0 \leq r^{\prime} \leq r$ being the content of strings, and

$$
\mathcal{L}=\left\{L_{l v} \mid l=1,2, \ldots, v=1,2, \ldots, m_{l}\right\}
$$

the set of all riggings of strings which enter the content $\nu$. The set

$$
R C(N, r)=\bigcup_{r^{\prime}=0}^{r} \bigcup_{\nu \vdash r^{\prime}} z(\nu)
$$

of all rigged strings which emerge from such paths has the cardinality

$$
|R C(N, r)|=\left(\begin{array}{c}
N \\
r
\end{array}\right)=\left|Q^{(r)}\right|
$$

which coincides with the dimension of the space $\mathcal{H}^{(r)}$ of quantum states of $r$ Bethe pseudoparticles. Moreover, each subset

$$
R C S\left(N, r^{\prime}\right)=\bigcup_{\nu \vdash r^{\prime}} z(\nu)
$$


has the cardinality

$$
\left|R C S\left(N, r^{\prime}\right)\right|=\operatorname{dim} \Delta^{\left\{N-r^{\prime}, r^{\prime}\right\}}=\left|S Y T\left\{N-r^{\prime}, r^{\prime}\right\}\right|
$$

which is equal to the dimension of the intersection

$$
\mathcal{H}^{(r)} \cap \mathcal{H}^{\left\{N-r^{\prime}, r^{\prime}\right\}}
$$

of the space $\mathcal{H}^{(r)}$ with the sector $\mathcal{H}^{\left\{N-r^{\prime}, r^{\prime}\right\}}$.

We proceed to describe the bijection $\rho=R S K K R: Q^{(r)} \rightarrow R C(N, r)$ of magnetic configurations entering the classical configuration space $Q^{(r)}$ of the system of $r$ Bethe pseudoparticles to the set $R C(N, r)$ of the corresponding rigged strings, i.e. the set of labels of exact BA eigenfunctions. It is evident that such a bijection can be established by local adjustment of a single-node spin $f(j)$ of a magnetic configuration $f \in \tilde{2}^{\tilde{N}}$ to the corresponding step $p(j)$ of the path $p$ which defines the rigged string $\nu \mathcal{L}=R S K K R(f)$. To this end, we introduce the notion of a compensated subword of a magnetic configuration $f$, treated as a word of the length $N$ in the alphabet $\tilde{2}$ of spins. Namely, a compensated subword $v$ of $f$ is a sequence of consecutive letters of $f$ (i.e. $f=a v b$; one of subwords $a$ and $b$ could be empty) such that (i) its weight $w(v)=\left(w_{-}(v), w_{+}(v)\right)$, with $w_{-}(v)$ and $w_{+}(v)$ being the multiplicity of occurrences of the letter "-" and "+" in the word $v$, respectively, satisfies $w_{-}(v)=w_{+}(v)$, (ii) $w_{-}\left(v^{\prime}\right)>w_{+}\left(v^{\prime}\right)$ for an arbitrary initial subword $v^{\prime}$ of $v$ (i.e. $v=v^{\prime} v^{\prime \prime}$ ). According to this definition, a compensated subword $v$ corresponds to a multipyramid if its letters "-" and "+" are related to the step up and down, respectively. Next, one observes that a magnetic configuration $f$ can be uniquely represented as the product

$$
f=u_{1} v_{1} u_{2} v_{2} \ldots u_{a} v_{a} u_{a+1}
$$

of compensated subwords $v_{1}, v_{2}, \ldots, v_{a}$, mediated by other subwords $u_{1}, u_{2}, \ldots, u_{a}, u_{a+1}$ (some of the latter could be empty), such that the subword

$$
u(f)=u_{1} u_{2} \ldots u_{a} u_{a+1}
$$

has no letter "+" having a letter "-" to the left of it. Now, the RSKKR bijection is established by defining the path $p(f)=\left(p_{1}(f), p_{2}(f), \ldots, p_{N}(f)\right)$, corresponding to the word $f$ given by Eq. (43) as

$$
p_{j}(f)=\left\{\begin{aligned}
\text { up } \quad \text { if } f(j) & =\text { "- " } \\
\text { or if } f(j) & =\text { "+" and } f(j) \in u(f), \\
\text { down if } \quad f(j) & =\text { "+" and } f(j) \in v(f),
\end{aligned}\right.
$$

with

$$
v(f)=v_{1} v_{2} \ldots v_{a} .
$$

Thus each compensated subword $v_{i}, i=1,2, \ldots, a$, is the preimage of a multipyramid on the path $p(f)$ and each subword $u_{i}, i=1,2, \ldots, a+1$, corresponds to a piece of the sea of holes. The nodes occupied by Bethe pseudoparticles, i.e. such that $f(j)=$ "-", yield all the steps up in the path $f(j)$, whereas those with vacuum, i.e. $f(j)=$ "+", yield either the step "up" when they belong to the sea of holes $u(f)$, or the step "down" when they are bound to a string. 
In this way, each magnetic configuration $f$ is mapped to a definite rigged string configuration $\nu \mathcal{L}=\rho(f)$. In Figs. 1 and $3, f$ is displayed below the $j$-axis on the diagram of a path $p(f)$, and $\nu \mathcal{L}$ to the right of this diagram, with riggings $L_{l v}$ inside the row corresponding to the string $(l, v)$, and the number of $l$-holes $P_{l}$ to the right of the $l \times m_{l^{-}}$ rectangle representing the $l$-strings in the content $\nu$.

The bijection $\rho$ is the composition of the famous RS algorithm which in our case yields the bijection

$$
R S: Q^{(r)} \rightarrow \bigcup_{r^{\prime}=0}^{r} S Y T\left(N-r^{\prime}, r^{\prime}\right) \times W T\left(N-r^{\prime}, r^{\prime}\right),
$$

and the KKR bijection

$$
K K R: S Y T\left(N-r^{\prime}, r^{\prime}\right) \times W T\left(N-r^{\prime}, r^{\prime}\right) \rightarrow R C S\left(N, r^{\prime}\right) \times W T\left(N-r^{\prime}, r^{\prime}\right) .
$$

The RS algorithm maps each magnetic configuration $f \in Q^{(r)}$ to the pair $(y, t)$ of standard Young and Weyl tableaux of one of the shapes $\left\{N-r^{\prime}, r^{\prime}\right\}, 0 \leq r^{\prime} \leq r$ in accordance with the Weyl duality. Then the KKR bijection maps this pair to $(\nu \mathcal{L}, t)=\rho(f)$. The resulting rigged string configuration $\nu \mathcal{L}$ can be described by saying that the path $p=$ $\left(p_{1}, p_{2}, \ldots, p_{N}\right)$ corresponding to the Young tableau $y$ is given by the formula

$$
p_{j}=\left\{\begin{array}{c}
\text { up } \\
\text { down }
\end{array}\right\} \text { if } j \text { is in the }\left\{\begin{array}{c}
\text { first } \\
\text { second }
\end{array}\right\} \text { row of } y .
$$

5. The geography of strings. The classical configuration space $Q^{(r)}$ of the system of $r$ Bethe pseudoparticles, i.e. the set of all positions $\mathbf{j}=\left(j_{1}, j_{2}, \ldots, j_{r}\right) \in Q^{(r)}$ of this system, is equipped with a natural geometry imposed by the action of the Heisenberg Hamiltonian $\hat{H}$ (Eq. (23)) and the translation group $C_{N}$. The first defines a local Cartesian coordinate system which is $r$-dimensional in the generic case when Bethe pseudoparticles are all remote, and $F$-dimensional when there are $F$ islands of consecutive nodes occupied by these pseudoparticles, $1 \leq F \leq r$. Orbits of the second action define locally the main diagonal of this Cartesian coordinate system, and globally they form loops which imply a curvature in $Q^{(r)}$.

Existence of the RSKKR bijection between the classical configuration space $Q^{(r)}$ and the corresponding set $R C(N, r)$ of rigged string configurations which classify exact BA eigenstates, impose an additional structure on the set $Q^{(r)}$ which we refer to as the geography of rigged strings.

To get an idea how rigged strings are distributed over the classical configuration space, we present in some detail the process of traveling of a single $l$-string,

$$
\nu \mathcal{L}=\underbrace{L}_{l} N-2 l
$$

along the corresponding $C_{N}$-orbit, i.e. the set

$$
\Theta_{l}=\left\{|j\rangle=A\left(C_{N}\right)^{j-1}|\underbrace{-\ldots-}_{l} \underbrace{+\ldots+}_{N-l}\rangle \mid j \in \tilde{N}\right\} .
$$

More formally, we define the mapping $\mathcal{K}_{N}=R C(N, r) \rightarrow R C(N, r)$ by requiring the 
diagram

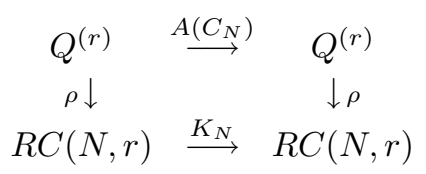

to be commutative, and study the image of the orbit $\Theta_{l}$ under this mapping. Clearly, $\mathcal{K}_{N}$ is the push-forward of the shift $A\left(C_{N}\right)$ from the classical configuration space $Q^{(r)}$ to the set $R C(N, r)$ of rigged strings. We get

$$
\rho(|j\rangle)= \begin{cases}\underbrace{\underbrace{j-1}_{l^{\prime}} N-2 l}_{l} & \text { for } 1 \leq j \leq N-2 l+1 \text { (regime a), } \\ \emptyset & \text { for } N-2 l+2 \leq j \leq N-l \text { (regime b), } \\ \underbrace{\underbrace{0} N-2 l^{\prime}}_{l^{\prime}} N-2 l^{\prime} & \text { for } N-l+2 \leq j \leq N \text { (regime d), }\end{cases}
$$

where

$$
l^{\prime}= \pm(N+1-l-j)
$$

with the upper and lower sign applied to the regime $b$ and $d$, respectively. Clearly, the orbit $\Theta_{l}$ constitutes the $F=1$-dimensional boundary of the classical configuration space $Q^{(l)}, l \leq N / 2$. The formula (53) and Fig. 5 imply a decomposition of this boundary into four regimes, with the regime a corresponding to travel of the $l$-string from the left rigging $L=0$ to the right $L=P_{l}=N-2 l$. The rest of the orbit $\Theta_{l}$ is no longer any preimage of the $l$-string, but rather some $l^{\prime}$-strings of a shorter length $l^{\prime}<l$. The regime $b$ corresponds to edge strings located at the right boundary of the (open) ring $\tilde{N}$, with the maximal rigging $L=P_{l^{\prime}}$, whereas the regime d to those at the left boundary, with the minimal rigging $L=0$. The regime c, corresponding to a single magnetic configuration, with all $l$ Bethe pseudoparticles at the end of the open chain $\tilde{N}$, corresponds to the state in $\mathcal{H}^{(r)}$, degenerated with the ferromagnetic vacuum state $|++\ldots+\rangle$ in energy. Clearly, magnetic configurations within the regime a correspond to the heighest weight exact BA eigenstates, with $S=N / 2-l$, and all Bethe pseudoparticles bound within the $l$-string, whereas the rest of the orbit $\Theta_{l}$ corresponds to lower weights, $S=N / 2-l^{\prime}$ with $l-l^{\prime}$ free Bethe pseudoparticles, located at the right side of the sea of holes.

Such analysis, applied above to the boundary $F=1$ of the classical configuration space, can be easily extended to any region of $Q^{(r)}$. It is clear from the considerations above that the string configuration $\nu$ of the image $\nu \mathcal{L}=\rho(f)$ of the magnetic configuration $f$ under the RSKKR bijection is essentially determined by the structure

$$
\xi=\left(\xi_{1}, \xi_{2}, \ldots, \xi_{F}\right), \sum_{a=1}^{F} \xi_{a}=r
$$




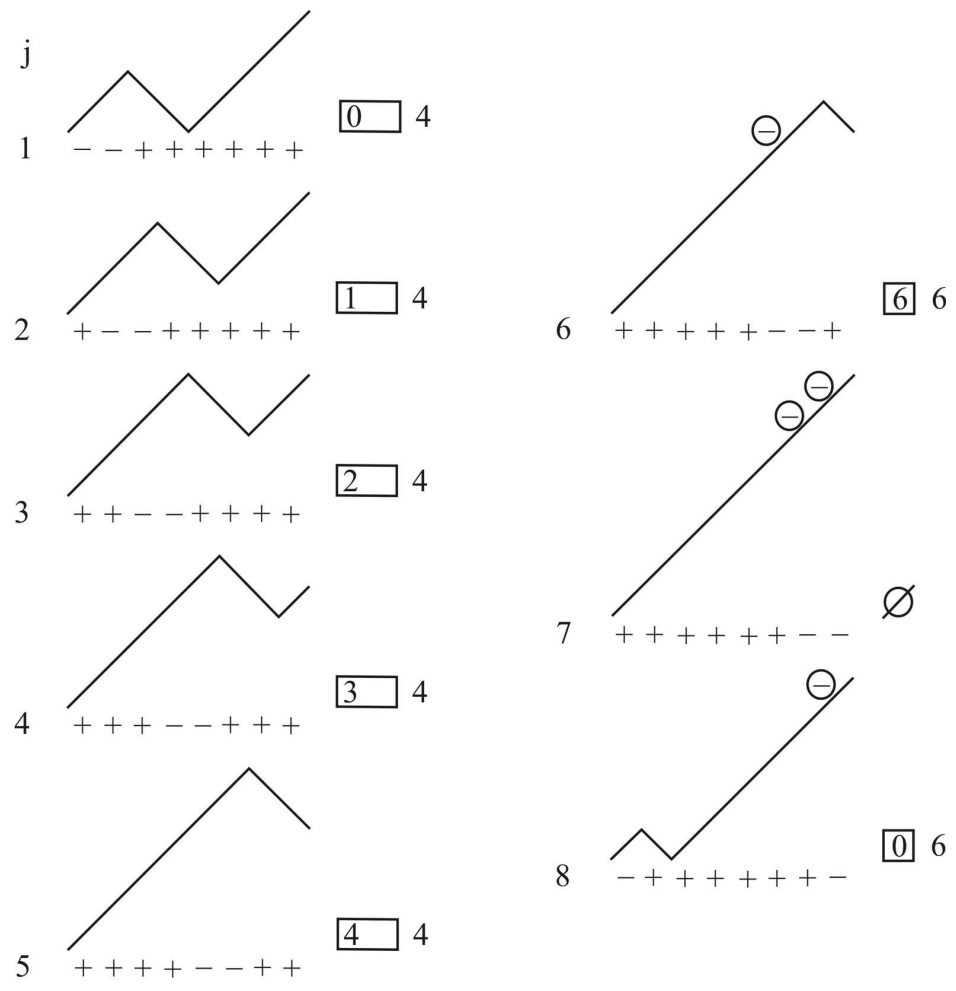

Fig. 5. Travel of the 2-string on the (open) chain for $N=8, r=l=2$

of islands of spin deviations in $Q^{(r)}$, whereas the appropriate riggings depend upon specific distribution of these islands on the sea of nodes occupied by the spin "+". In all those cases when each Bethe pseudoparticle has its own "+" counterpart to the right, we have, with a little abuse of notation,

$$
\nu=\xi
$$

after appropriate rearrangement of arguments of $\xi$ such that $\nu$ should be a standard partition. Then, in particular, $m_{l}$ is the number of islands of the length $l$. Such cases correspond to the highest weight states with $S=N / 2-r$. In other cases, $r-r^{\prime}$ Bethe pseudoparticles remain unbound into strings, and are thus located at the right side of the sea of holes. Then $S=N / 2-r^{\prime}$.

The total number of strings in a given exact BA eigenfunction is

$$
q(\nu \mathcal{L})=\sum_{l} m_{l}
$$

Let $Q^{(r, F)} \subset Q^{(r)}$ be the $F$-dimensional boundary of $Q^{(r)}, 1 \leq F \leq r$. It follows from the action of the mapping $\mathcal{K}_{N}$ on the RSKKR image of any $C_{N^{-}}$orbit in $Q^{(r)}$ that

$$
q(\rho(f)) \in\{F, F-1\} \text { for } f \in Q^{(r, F)} .
$$

This number is simply equal to that of cuspidal maxima inside the corresponding path 
$p(f)$, which, in turn, is equal to $F$ when each island contributes to a string of length at least 1 , or $F-1$ when this island forms a terminal subword of $f$ and thus is not related to a string. In this way, RSKKR bijection associates each string with its parent island of spin deviations, which can be traced by appropriate cuspidal maximum on the path $p(f)$. This tracing, however, cannot be done when consecutive maxima have the same height on the path, since then the correspondence between pyramids and strings is not unique (cf., e.g., the third path in Fig. 3).

Clearly, the length of the string originating from an island $\xi_{a}$ cannot exceed the size of this island, but may be smaller (due to transgression of the island through the boundary of the open chain $\tilde{N}$ along an appropriate $C_{N \text {-orbit), so that }}$

$$
0 \leq l \leq \xi_{a}
$$

Also, a string cannot arise from two or more separated islands. In particular, the generic part $Q^{(r, r)}(F=r)$ of the classical configuration space yields only 1-strings, that is,

$$
\rho\left(Q^{(r, r)}\right) \subset \bigcup_{r^{\prime}=1}^{r} z\left(1^{r^{\prime}}\right) .
$$

We present here a small, but reasonably typical example of geography of strings for $N=8$ and the classical configuration space

$$
Q^{(3)}=Q^{(3,1)} \cup Q^{(3,2)} \cup Q^{(3,3)},
$$

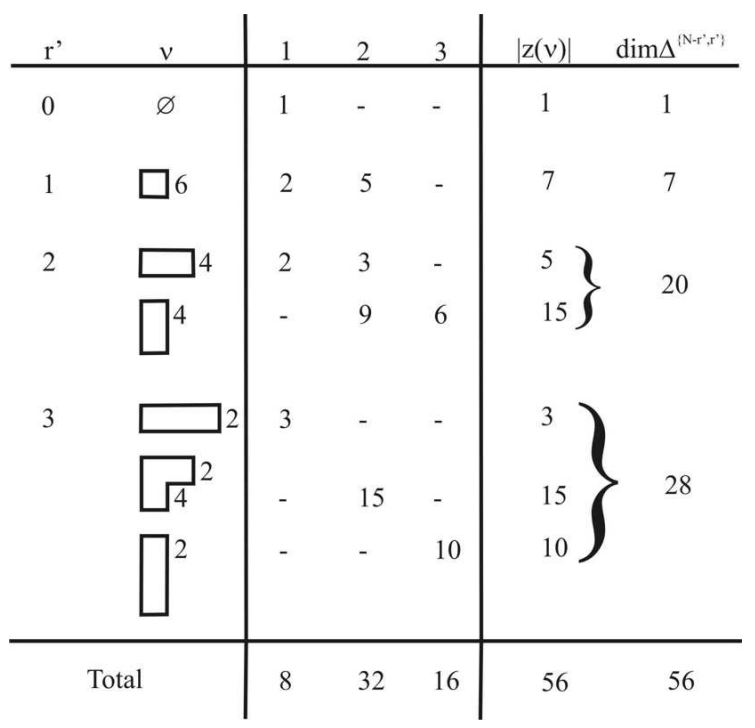

Table 1. Distribution of strings over the classical configuration space $Q^{(3)}$ for $N=8$. Each row describes numbers of rigged string configurations with a given content $\nu$ over the sets $Q^{(3, F)}$. The detailed distribution is shown in Fig. 6. 


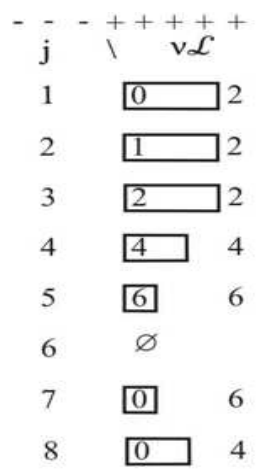

a) The boundary $F=1$

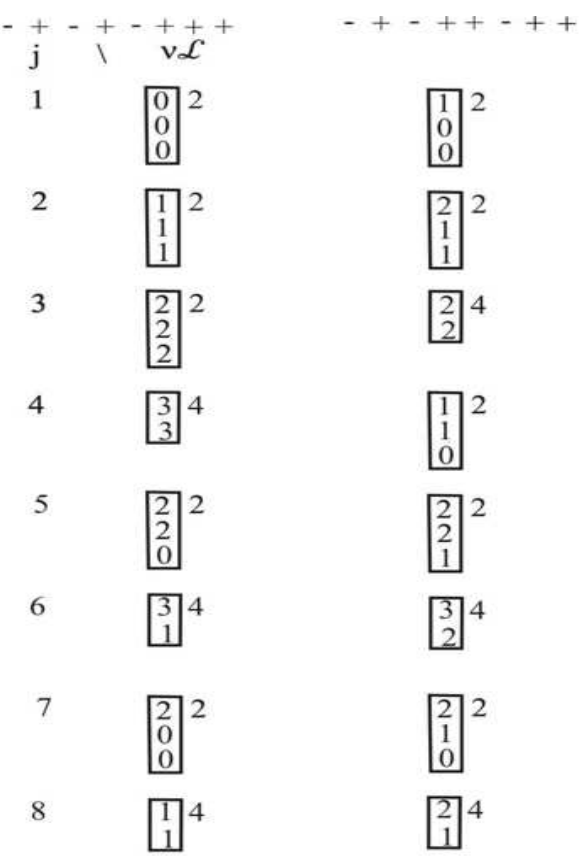

c) The generic part $\mathrm{F}=3$

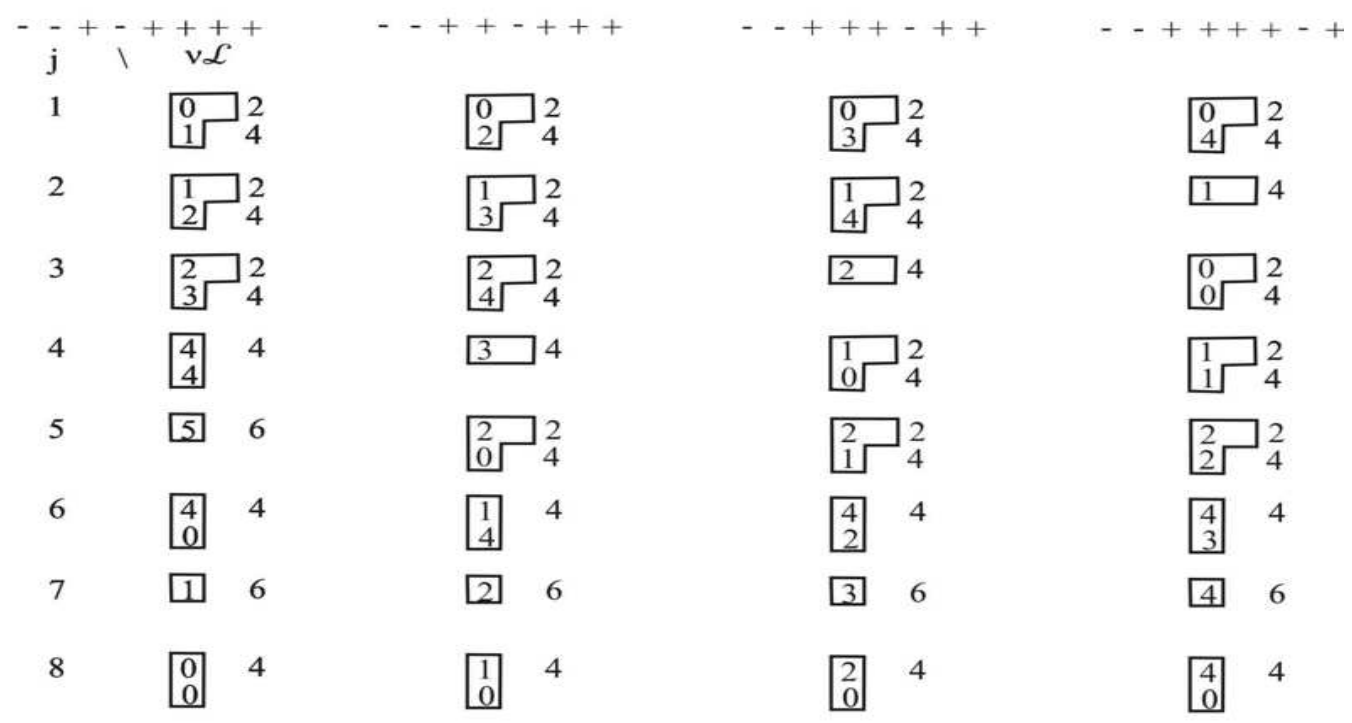

b) The boundary $\mathrm{F}=2$

Fig. 6. The geography of $l$-strings on the classical configuration space $Q^{(3)}$ for $N=8$. $C_{8}$-orbits are arranged in columns. The initial magnetic configuration is displayed at the top of an orbit. 
with the cardinalities $56=8+32+16$ (cf. Fig. 6 and Table 1). The 3 -strings, i.e. maximally bound states of the system of 3 Bethe pseudoparticles, are found only in the one-dimensional boundary $Q^{(3,1)}$. This boundary encloses also two 2-strings, two 1 -strings, and the vacuum configuration $\emptyset$, but the total number of strings in each $\nu \mathcal{L}$ is here $q(\nu \mathcal{L})=1$. It is a constatation of the fact that a single island yields at most one string. The two-dimensional boundary $Q^{(3,2)}$ is the homeland for four string contents, $\nu \in\left\{\{1\},\{2\},\left\{1^{2}\right\},\{21\}\right\}$, which is also consistent with the available structure $(2+1)$ of islands. The number of strings is 1 or 2, in accordance with Eq. (58). The generic, threedimensional part $Q^{(3,3)}$ yields only strings of length $1, \nu \in\left\{\left\{1^{2}\right\},\left\{1^{3}\right\}\right\}$. The number of strings is $q(\nu \mathcal{L})=3$ when all three islands (Bethe pseudoparticles in this case) are inside the open chain $\tilde{N}$, and $q(\nu \mathcal{L})=2$ for cases when one of these islands is at the end (i.e., for $\nu \mathcal{L}=\rho(f)$ such that $f(N)=$ "-").

6. Conclusions. We have presented here the composition of two famous combinatorial bijections: RS and KKR, applied to the magnetic Heisenberg chain for the spin $1 / 2$. This RSKKR bijection maps the set $\tilde{2}^{\tilde{N}}$ of all magnetic configurations to the set $R C(N)$ of all exact BA eigenstates, labeled by rigged strings. This bijection has a natural restriction to the set $Q^{(r)}$ of all magnetic configurations with $r$ reversed spins, and the latter set is shown to have an interpretation of the configuration space (in the meaning used in mechanics) for a classical counterpart of the (quantum) Heisenberg model of a magnetic chain. This shows that the RSKKR bijection plays the role of defining the geography of rigged strings on $Q^{(r)}$. We have presented here this geography in some detail. In particular, we point out that rigged strings have their origin in the structure of islands of spin deviations (or of Bethe pseudoparticles) on the classical configuration space for a given magnetic configuration.

\section{References}

[1] H. Bethe, Z. Physik 71 (1931), 205.

[2] R. Baxter, Exactly Solvable Models in Statistical Mechanics, Academic Press, New York, 1982 .

[3] M. Gaudin, La fonction d'onde de Bethe, Masson, Paris, 1983.

[4] L. D. Faddeev and L. A. Takhtadzhyan, Zap. Nauch. Semin. LOMI 109 (1981), 134 (in Russian); English transl.: J. Sov. Math. 24 (1984), 241.

[5] S. V. Kerov, A. N. Kirillov and N. Yu. Reshetikhin, Zap. Nauch. Semin. LOMI 155 (1986), 50 (in Russian); English transl.: J. Sov. Math. 41 (1988), 916.

[6] R. Robinson, Amer. J. Math. 60 (1938), 745.

[7] R. Schensted, Canad. J. Math. 13 (1961), 179.

[8] G. Knuth, Pacific J. Math. 34 (1970), 709.

[9] A. Lascoux, B. Leclerc, J. Y. Thibon, The Plactic Monoid, in: Algebraic Combinatorics on Words, M. Lothaire (ed.), Univ. Press, Cambridge, 2001.

[10] T. Lulek, B. Lulek, D. Jakubczyk and P. Jakubczyk, Physica B: Condensed Matter 382 (2006), 162-180.

[11] B. Lulek and T. Lulek, Rep. Math. Phys. 38 (1996), 267-271. 
[12] B. Lulek and D. Jakubczyk, CEJP 1 (2003), 132.

[13] H. Weyl, Gruppentheorie und Quantummechanik, Hirzel, Leipzig, 1931; English transl.: Dover, New York, 1950.

[14] S. O. Warnaar, J. Stat. Phys. 82 (1996), 657.

[15] T. A. Welsh, Memoirs of the AMS 175 (2005), 827.

[16] T. A. Welsh, J. Phys. Conference series 30 (2006), 119. 
